\title{
Etude de quelques facteurs de variation de la digestibilité in vitro \\ Application à un foin traité ou non à la soude
}

\author{
L. ISTASSE, C. VAN EENAEME, O. LAMBOT, M. GIELEN \\ et J.M. BIENFAIT \\ Faculté de Médecine vétérinaire de l'Université de Liège \\ 45 , rue des Vétérinaires, 1070 Bruxelles (Belgique)
}

\section{Résumé}

La technique de Tilley et TerRy et celle des sachets de nylon ont été utilisées pour estimer la digestibilité d'un foin traité ou non à la soude, en exploitation. Les concentrations utilisées sont de 3 et $6 \%$ de $\mathrm{NaOH}$ par rapport à l'aliment brut. Douze taurillons recevant trois rations qui différaient entre elles par les proportions des aliments distribués ont été utilisés conme donneurs de liquide du rumen et porteurs des sachets de nylon.

Ces techniques de digestibilité permettent de mettre en évidence un effet du traitement à la soude mais elles surestiment l'efficacité de ce traitement lorsqu'on se réfère aux valeurs déterminées sur l'animal. C'est avec une teneur en soude de $3 \%$ que l'on a enregistré la plus haute digestibilité «in vitro». Les rations utilisées n'ont pas eu d'influence au niveau des estimations des coefficients de digestibilité «in vitro» mais on a observé des variations significatives entre les animaux.

\section{Introduction}

L'appréciation de la valeur énergétique des aliments est fortement liée à la détermination de leur digestibilité. Les mesures faites sur animaux nécessitent des installations complexes et des quantités importantes d'aliments à tester ; elles sont donc très coûteuses. Pour obvier à ces difficultés, de nombreux chercheurs ont mis au point des techniques de laboratoire qui permettent d'obtenir une estimation correcte des coefficients de digestibilité pour autant qu'il y ait une bonne corrélation entre les valeurs «in vivo» et «in vitro».

Il est donc intéressant d'étudier certains paramètres susceptibles de modifier les résultats «in vitro». Comme les techniques de TILLEY et TERRY (1963) et des sachets de nylon sont toutes deux basées sur la fermentation d'un échantillon d'aliment dans du liquide du rumen on a voulu vérifier l'influence des rations distribuées aux animaux sur l'activité enzymatique et la composition de ce milieu biologique très complexe. C'est pourquoi, les déterminations ont été réalisées avec des taureaux recevant des rations qui différaient par les proportions des aliments utilisés et la teneur en matières azotées $(\mathrm{N} \times 6,25)$. Les expériences de digestibilité ont porté sur un foin traité ou 
non à la soude, en exploitation, par une machine danoise. Les digestibilités « in vivo » de ces foins avaient été déterminées antérieurement sur taureaux (VAN EENAEME et al., $1981)$.

\section{Matériel et méthode}

La technique de Tilley et Terry s'effectue en deux étapes : une fermentation anaérobie de l'aliment dans un mélange de liquide du rumen et de tampon suivie d'une attaque par une solution de pepsine et d'acide chlorhydrique. La première étape simule l'action de la digestion microbienne dans le réseau-rumen. La seconde imite l'attaque enzymatique de l'aliment dans la caillette et le début de l'intestin. Van HeE \& CarLIER (1970) donnent une description détaillée de cette technique.

Les sachets de nylon ont été réalisés selon la méthode rapportée par AERTS et a!. (1976). Les sachets, aux dimensions internes de $6 \times 7 \mathrm{~cm}$, sont confectionnés dans un tissu de nylon à mailles très fines $(50 \mu \mathrm{m}$ ) (Nylon Type Monodur PA 50 : Heleine et Cavenaile - Bruxelles). Ils contiennent $2 \mathrm{~g}$ d'aliment dont on veut déterminer la digestibilité et sont introduits dans le rumen par l'intermédiaire d'une canule. Après un séjour de 48 heures, les sachets sont retirés du rumen, lavés sous eau courante et placés dans une solution de pepsine et d'acide chlorhydrique à $39^{\circ} \mathrm{C}$ pour une nouvelle durée de 48 heures. Cette solution est identique à celle utilisée dans la technique de TILley et TERry.

Après cette seconde attaque, les sachets sont à nouveau rincés et séchés. La digestibilité de la matière sèche est obtenue par différence entre le poids de l'échantillon et celui du résidu. La détermination de la teneur en cendres permet de calculer la digestibilité de la matière organique.

Les déterminations «in vitro» furent réalisées sur douze taurillons faisant l'objet d'une expérience d'engraissement. Les animaux étaient munis d'une canule du rumen et répartis au hasard en trois groupes auxquels furent distribués trois rations à teneur différente en matières azotées. Ces rations étaient constituées de pulpes séchées ce betteraves sucrières, orge aplatie, tourteau de soja et foin de graminées (tableau 1).

Le tableau 2 résume quelques caractéristiques relatives au liquide du rumen prélevé chez les animaux avant le repas du matin, pour les trois niveaux protéiques.

Le schéma expérimental comportait trois blocs (rations) divisés en sous-traitements constitués par les trois types de foin à tester. Les analyses de variance ont été effectuées suivant un modèle à trois critères de classification partiellement hiérarchisés : 3 rations dont les proportions des aliments étaient différentes, 3 foins et 4 animaux par ration (DAGNELIE, 1970).

La digestibilité «in vivo» avait été déterminée suivant la technique décrite par SCHNEIDER \& Flatt (1975) sur d'autres jeunes taureaux de $300 \mathrm{~kg}$ qui recevaient 1,1 ou $2,2 \mathrm{~kg}$ des foins à tester ainsi que $2,2 \mathrm{~kg}$ d'un concentré protéique à $20 \mathrm{p}$. cent, $2,2 \mathrm{~kg}$ de pulpes séchées de betteraves sucrières et $0,1 \mathrm{~kg}$ de mélange minéral vitaminé. Le foin étudié a subi un traitement à la soude réalisé en exploitation par une machine danoise (Machine JF Type SP 2000 - JF Fabriken - J. Freudendahl A/S/Sonderborg Danemark). Ce traitement consiste en un hachage du foin suivi par la pulvérisation de 


\section{Tableau 1}

Rations distribuées aux taurillons ( $\mathrm{kg}$ par jour)

Teneurs en matières azotées et cellulose brute (\% de la matière fraîche de la ration)

Diets offered to young bulls ( $\mathrm{kg}$ per day)

Crude protein and fibre contents (\% of dietary fresh matter)

\begin{tabular}{|c|c|c|c|}
\hline \multirow{2}{*}{ Aliments (Ingredients) } & \multicolumn{3}{|c|}{$\begin{array}{l}\text { Rations à teneur en matières azotées } \\
\text { Crude protein level }\end{array}$} \\
\hline & $\begin{array}{l}\text { Faible } \\
\text { Low }\end{array}$ & $\begin{array}{l}\text { Moyenne } \\
\text { Medium }\end{array}$ & $\begin{array}{l}\text { Elevée } \\
\text { High }\end{array}$ \\
\hline Soja (Soybean) & 0,5 & 1,1 & 1,7 \\
\hline Orge $($ Barley $) \ldots \ldots \ldots \ldots \ldots \ldots \ldots \ldots \ldots$ & 3 & 2,2 & 2 \\
\hline Pulpes déshydratées de betteraves (Dried beet pulps) & 4 & 4,6 & 4,8 \\
\hline Foin $($ Hay $) \ldots \ldots \ldots \ldots \ldots \ldots \ldots \ldots \ldots \ldots \ldots \ldots$ & 1 & 1 & 1 \\
\hline $\begin{array}{c}\text { Complément minéral vitaminé (Minerals and vita- } \\
\text { mins) } \ldots \ldots \ldots \ldots \ldots \ldots \ldots \ldots \ldots \ldots \ldots \ldots \ldots \ldots \ldots \ldots\end{array}$ & 0,2 & 0,2 & 0,2 \\
\hline \multicolumn{4}{|l|}{ Teneurs (Contents) } \\
\hline Matières azotées $(\mathrm{N} \times 6,25)$ (Crude protein) $\ldots$ & 10,8 & 12,8 & 14,6 \\
\hline Cellulose brute (de Weende) (Weende crude fibre) & 15,5 & 15,9 & 15,8 \\
\hline
\end{tabular}

\section{TABLEAU 2}

Quelques caractéristiques du liquide du rumen prélevé avant le repas du matin Some characteristics of the rumen fluid sampled before the morning meal

\begin{tabular}{|c|c|c|c|}
\hline & \multicolumn{3}{|c|}{$\begin{array}{c}\text { Rations à teneur en matières azotées } \\
\text { Crude protein level }\end{array}$} \\
\hline & $\begin{array}{l}\text { Faible } \\
\text { Low }\end{array}$ & $\begin{array}{l}\text { Moyenne } \\
\text { Medium }\end{array}$ & $\begin{array}{l}\text { Elevée } \\
\text { High }\end{array}$ \\
\hline $\begin{array}{l}\text { Concentration totale en acides gras volatils } \\
\quad(\text { mmoles } / 1) \text {. } \ldots \ldots \ldots \ldots \ldots \ldots \ldots \ldots \ldots \ldots \\
\text { Total volatile fatty acid concentration }\end{array}$ & 80,8 & 83,0 & 88,3 \\
\hline $\begin{array}{l}\text { Proportion des A.G.V. (\% molaire) } \\
\text { V.F.A. proportion (molar } \% \text { ) }\end{array}$ & & & \\
\hline 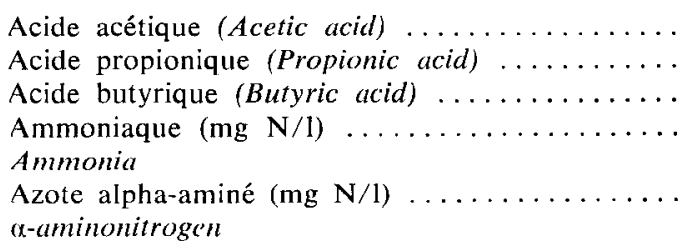 & $\begin{array}{l}65,1 \\
21,6 \\
12,7 \\
11,0 \\
\\
38,6\end{array}$ & $\begin{array}{l}64,6 \\
18,9 \\
16,0 \\
73,0 \\
\\
37,5\end{array}$ & $\begin{array}{l}65,9 \\
20,0 \\
13,9 \\
83,0 \\
\\
29,8\end{array}$ \\
\hline
\end{tabular}


la solution de soude à $33 \mathrm{p}$. cent. Deux teneurs ont été utilisées : 3 et $6 \mathrm{~kg}$ de soude par $100 \mathrm{~kg}$ de foin. Les brins de foin sont ensuite intimement mélangés avec la solution et envoyés par une soufflerie dans le local de stockage. Le tableau 3 donne la composition des foins étudiés. On notera une augmentation de la teneur en cendres totales supérieure aux deux niveaux de traitements à la soude; cette augmentation peut s'expliquer par le fait que la soude ajoutée est dosée sous forme de $\mathrm{Na}_{2} \mathrm{O}$ et/ou de $\mathrm{Na}_{2} \mathrm{CO}_{3}$ dans les cendres totales. En revanche, les concentrations en matières azotées et en cellulose brute restent constantes dans la matière organique.

\section{TABLEAU 3}

\section{Composition des foins étudiés}

Hay composition

\begin{tabular}{|c|c|c|c|}
\hline & $\begin{array}{c}\text { Foin } \\
\text { non traité } \\
\text { Untreated hay }\end{array}$ & $\begin{array}{c}\text { Foin traité } \\
\text { à } 3 \% \text { de soude } \\
\text { Hay treated } \\
\text { with } 3 \% \mathrm{NaOH}\end{array}$ & $\begin{array}{c}\text { Foin traité } \\
\text { à } 6 \% \text { de soude } \\
\text { Hay treated } \\
\text { with } 6 \% \mathrm{NaOH}\end{array}$ \\
\hline $\begin{array}{l}\text { Matière sèche (en \% du produit brut) } \ldots \\
\text { Dry matter (in \% of crude product) }\end{array}$ & 81,8 & 79,0 & 75,3 \\
\hline $\begin{array}{l}\text { Matière organique (en } \% \text { du produit brut) } \\
\text { Organic matter }\end{array}$ & 73,1 & 66,8 & 60,2 \\
\hline $\begin{array}{l}\text { Cendres (en } \% \text { de la M.S.) } \ldots \ldots \ldots \\
\text { Ash (in } \% \text { D.M.) }\end{array}$ & 10,6 & 15,4 & 20,1 \\
\hline $\begin{array}{l}\text { Matières azotées (en } \% \text { de la M.S.) .... } \\
\text { Crude protein }\end{array}$ & 11,7 & 11,0 & 10,5 \\
\hline $\begin{array}{l}\text { Cellulose brute (en } \% \text { de la M.S.) .... } \\
\text { Crude fibre }\end{array}$ & 34,5 & 32,7 & 30,8 \\
\hline
\end{tabular}

\section{Résultats}

Les coefficients de digestibilité «in vitro» et «in vivo» de la matière sèche, de la matière organique et de la cellulose brute sont rapportés au tableau 4 . Le traitement à la soude augmente de manière hautement significative la digestibilité du foin. Pour la matière organique, on enregistre des augmentations de 19,3 et 15,1 points avec la technique de TILley et TERRY et de 26,5 et 19,8 points avec la technique des sachets de nylon pour les foins traités à 3 et $6 \mathrm{p}$. cent de soude. L'amélioration de la digestibilité de la matière organique «in vivo » est moins élevée : respectivement 11,3 et 7,1 points. 
(2)

$2 \quad: \frac{0}{2}$

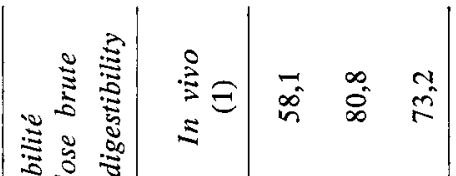

$\begin{array}{cc}2 & 8 \\ 2 & 3\end{array}$

$\stackrel{3}{\Xi}$

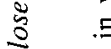

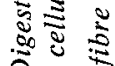

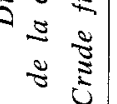

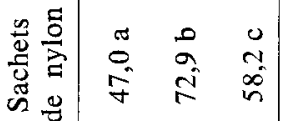

$\overrightarrow{8}$
0
0
0
0

$\begin{array}{lll}* & n & n \\ * & Z & Z \\ * & n & N \\ i n & 0 & i\end{array}$

$\stackrel{0}{3}$

ज.

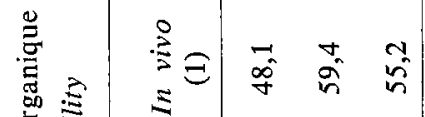

ป.

氙 范

I

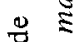

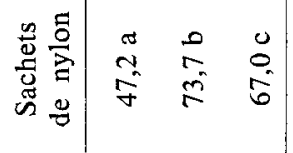

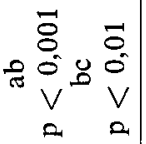

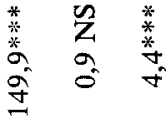

苋

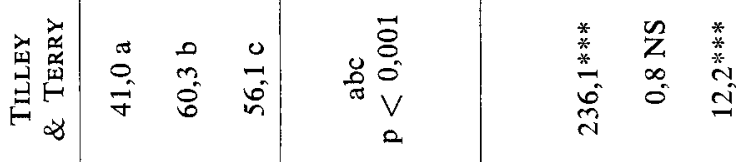

$\stackrel{\infty}{\bar{a}}$

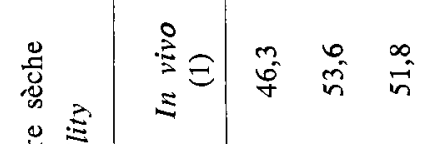

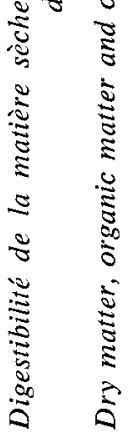

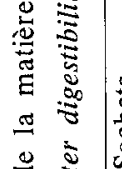

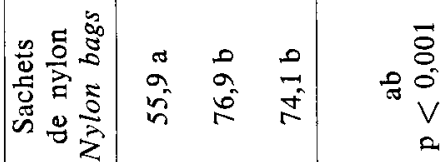

ษ

苋

总

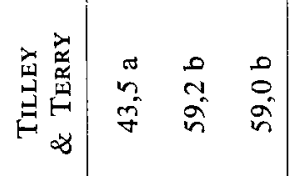

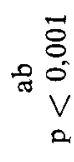

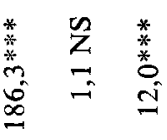

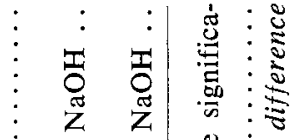

莺 离

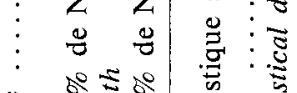

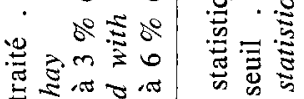

Е

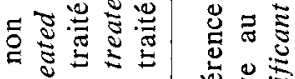

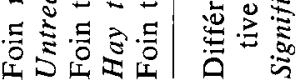

吉ま芒

山

द⿱ 口)

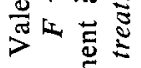

壳: : 


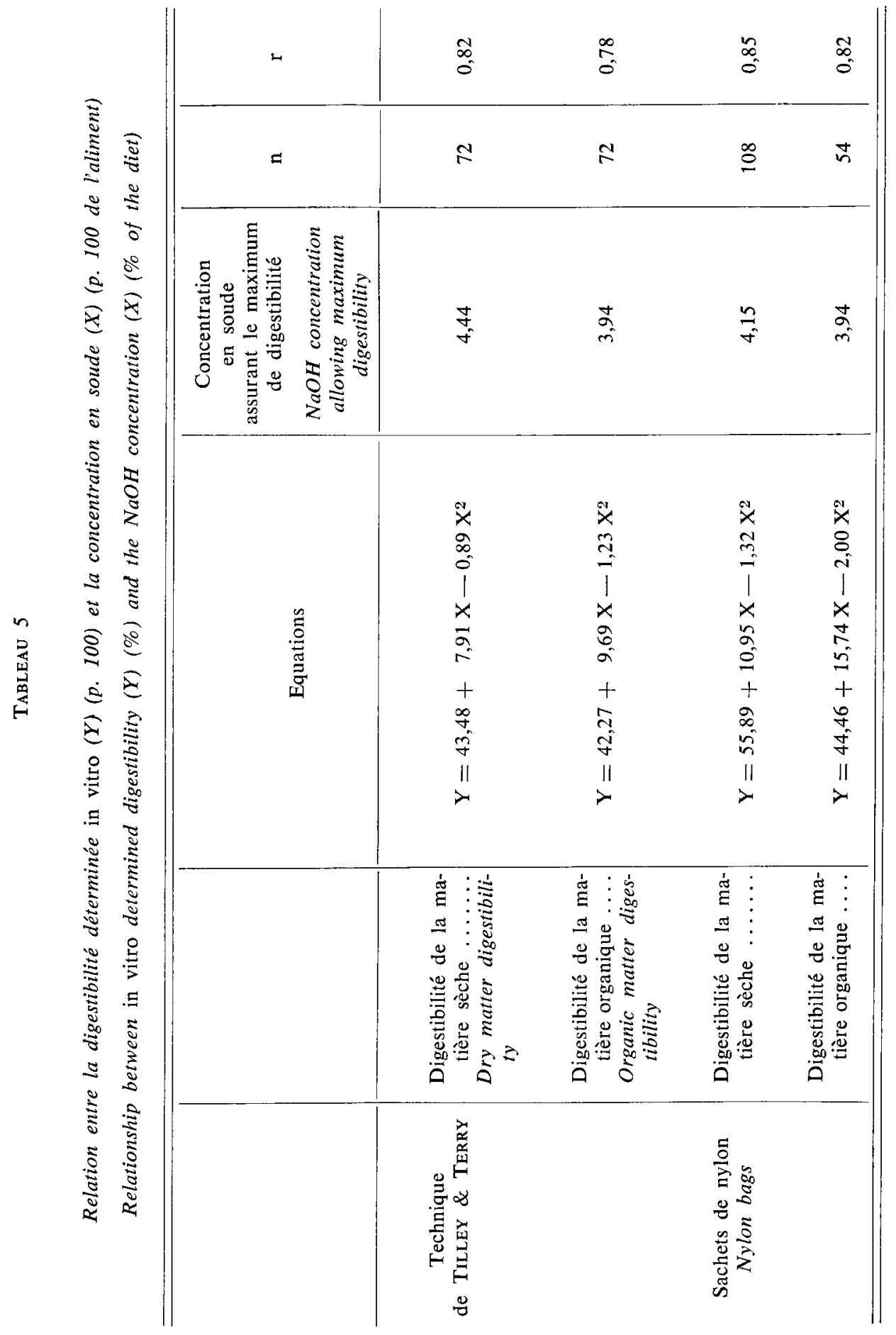




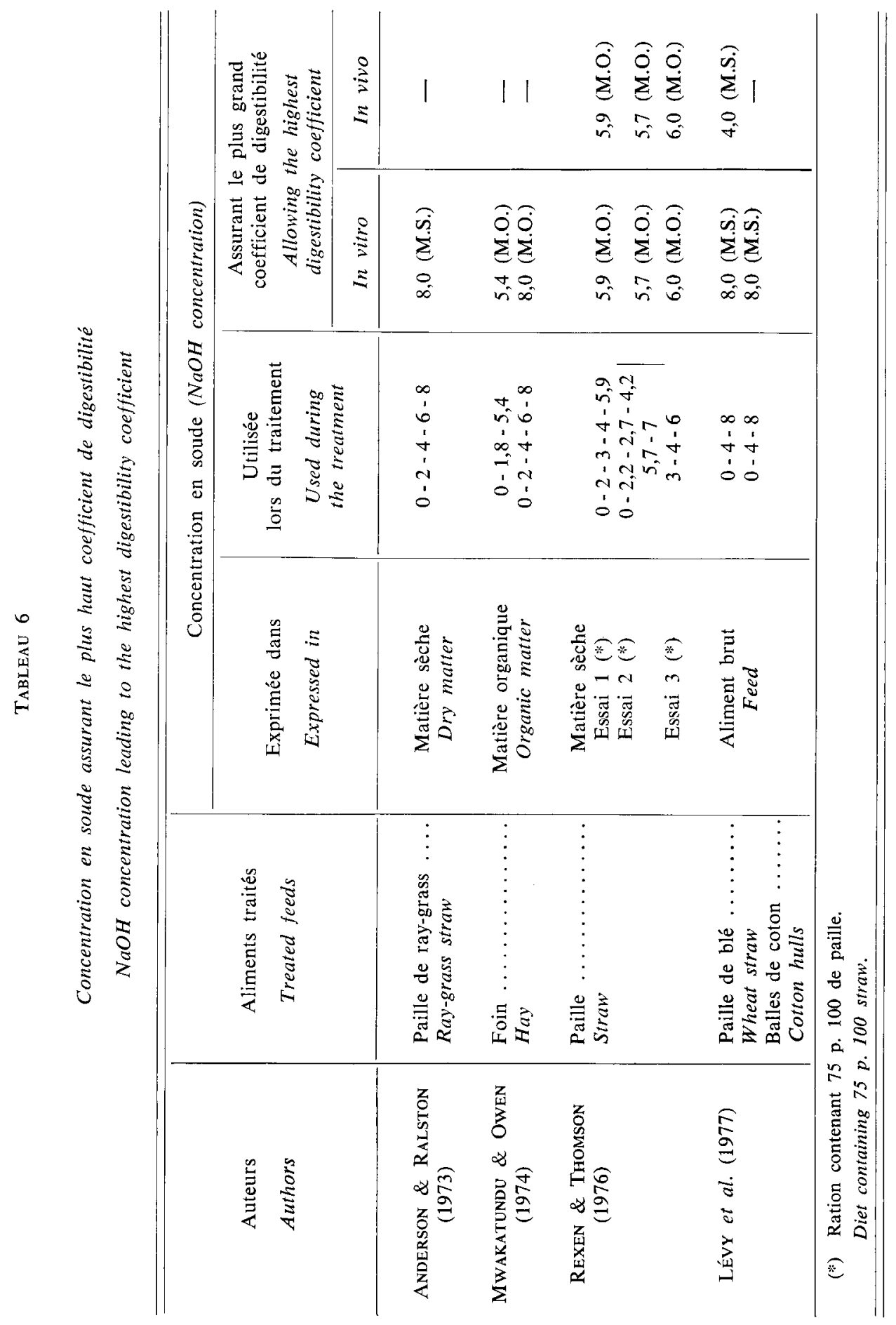


C'est à 3 p. cent de soude que l'on enregistre les valeurs de digestibilité les plus élevées aussi bien «in vivo» que «in vitro». Les valeurs observées permettent de calculer une équation de régression du second degré entre la concentration de soude (X) et les coefficients de digestibilité (Y). Ces équations figurent au tableau 5 ainsi que la concentration en soude qui, d'après ces équations, assure le maximum de digestibilité. On notera la bonne concordance de l'optimum pour la digestibilité de la matière organique suivant les deux techniques «in vitro».

D'après l'analyse de variance, il n'est pas possible de mettre en évidence une influence des proportions des aliments constitutifs des trois régimes sur les estimations des coefficients de digestibilité. En revanche, on a enregistré des différences individuelles très hautement significatives entre les coefficients de digestibilité de la matière sèche ou de la matière organique obtenus sur les animaux recevant un même régime. Pour la digestibilité de la cellulose brute mesurée par les sachets de nylon, les différences individuelles n'atteignent pas le seuil de signification.

\section{Discussion}

En comparant entre eux les coefficients obtenus par les deux méthodes de laboratoire, on constate que les valeurs mesurées par la technique des sachets de nylon sont systématiquement supérieures aux coefficients obtenus par la technique de Tilleey et Terry. Des constatations identiques ont été rapportées par CoOMBE, DINIUS \& WheEler (1979) à propos de la digestibilité de la matière sèche de paille traitée à la soude : après 48 heures d'incubation, les auteurs obtiennent, avec la technique des sachets de nylon, des coefficients supérieurs de 4 points aux valeurs mesurées par la technique de TILLEY et TERRY. L'obtention de valeurs plus élevées avec la technique des sachets de nylon peut provenir d'une différence dans l'établissement de l'état d'équilibre entre le substrat (aliments), les réactifs (enzymes) et les produits de la réaction (métabolites). Dans la technique des sachets, une faible quantité d'aliments est mise en incubation dans une grande masse de réactifs, les produits de la réaction sont éliminés en continu de telle sorte que l'équilibre est fortement déplacé vers la production des métabolites. Dans la technique de TiLley et Terry, seuls les gaz peuvent s'échapper; après quelques temps de fermentation, une accumulation de métabolites freine la réaction.

La différence dans la mesure de l'efficacité du traitement à la soude selon les déterminations «in vitro» et «in vivo» est due au fait que les techniques « in vitro» semblent d'une part sous-estimer la digestibilité du foin non traité et d'autre part surestimer celle du foin traité. ReXen \& Thomson (1976), ainsi que Berger, KLopFENSTEIN \& BRITTON (1979) ont enregistré des observations similaires avec de la paille et des rafles de maïs traitées à la soude. La faible amélioration de la digestibilité «in vivo» du foin traité à la soude est vraisemblablement attribuable à la proportion élevée de concentré dans la ration qui entraîne une diminution de l'activité cellulolytique des microorganismes du rumen. Dulphy (1979) montre qu'avec des pailles traitées ou non à la soude, une proportion élevée de concentrés dans la ration abaisse le niveau de digestibilité.

La quantité de soude qui permet d'obtenir une digestibilité maximale est de l'ordre de 4 p. cent. Le tableau 6 permet de comparer cette valeur à celles enregis- 
trées par d'autres auteurs. De ce tableau ainsi que de nombreuses données concernant le traitement des pailles à la soude (WILson \& Pigden, 1964 ; SingH \& JACKson, 1971), il apparaît que la digestibilité «in vitro» s'élève lorsqu'on augmente la teneur en soude. En revanche «in vivo», on observe que les améliorations de la digestibilité et de l'ingestibilité s'amenuisent lorsqu'on dépasse des concentrations de plus de 4 p. cent de NaOH (Ali, Mason \& Waagepetersen, 1977). La diminution de la digestibilité du foin traité à $6 \mathrm{p}$. cent par rapport à celle mesurée à $3 \mathrm{p}$. cent serait due à des teneurs élevées en produits peu digestibles provenant des réactions de MAILlaRD (VAN Eenaeme et al., 1981).

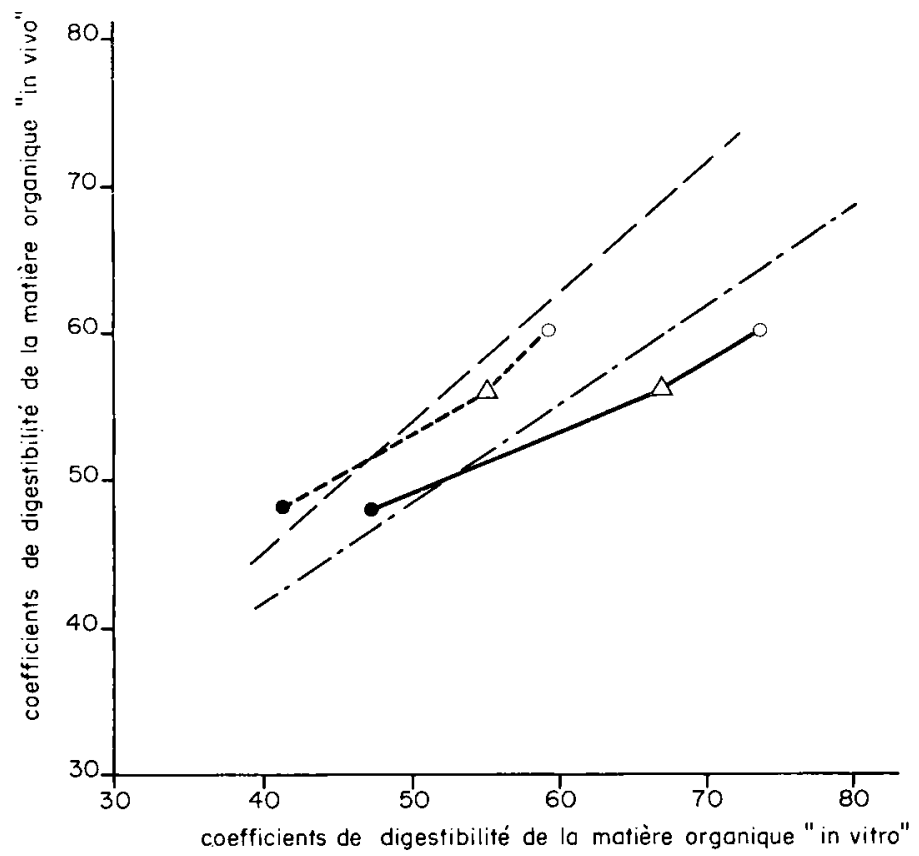

FIG. 1

Relation entre les coefficients de digestibilité de la matière organique mesurés « in vivo» et « in vitro»

Relationship between the digestibility coefficients of the organic matter measured «in vivo» and «in vitro»

- Foin non traité - Untrcated hay.

Foin traité à $3 \%$ de $\mathrm{NaOH}$ - Hay treated with $3 \% \mathrm{NaOH}$.

$\triangle$ Foin traité à $6 \%$ de $\mathrm{NaOH}-\mathrm{Hay}$ treated with $6 \% \mathrm{NaOH}$.

— Sachet de nylon - Nylon bag.

- . . . . - Tilley et Terry.

- - - AERTS et al. (1976) - $\mathrm{y}=13,47+0,69 \mathrm{x}$.

- - Cottyn, Van Hee, Carlier (1970) - y=10,04 +0,88 x.

$\mathrm{y}$ : digestibilité «in vivo» - «in vivo» digestibility.

$\mathrm{x}$ : digestibilité «in vitro» - «in vitro» digestibility : - bags (AERTS ot al.) - TILlEY et TERry (CotTyn et al.). 
La figure 1 (relation entre les résultats «in vivo» et «in vitro» de la digestibilité de la matière organique) résume les différentes constatations. Un certain parallélisme des deux tracés indique que les deux techniques de laboratoire mettent en évidence des variations de digestibilité semblables. Les valeurs observées dans l'expérience présente s'écartent peu de celles que l'on obtient en appliquant les équations, d'une part de AERTs et al. (1976) entre la digestibilité «in vivo» et la digestibilité en sachets pour des foins et d'autre part de CotTyn, Van HeE \& Carlier (1970) entre la digestibilité «in vivo» et la digestibilité déterminée par la technique de TILLEY et TERRY.

De nombreux auteurs se sont intéressés à l'influence des rations distribuées aux animaux sur les coefficients de digestibilité déterminés "in vitro». Comme le soulignent NiK-KhaH \& Tribe (1977), les résultats sont souvent contradictoires et pas toujours très concluants. Ces divergences s'expliquent par la variabilité des schémas expérimentaux utilisés.

Nous n'avons pas mis en évidence d'influence des proportions des aliments distribués sur les coefficients de digestibilité «in vitro». Ces rations, riches en amidon, hémicellulose, pectines et sucres solubles ne différaient entre elles que par leur teneur en matières azotées. Ces différences sont relativement faibles $(3,8 \mathrm{p}$. cent $)$ et n'ont pas entraîné de modifications importantes de l'activité digestive du liquide du rumen. En revanche, certains auteurs ont enregistré une influence marquée des rations à base de foins à teneur en matières azotées très différentes sur les déterminations de la digestibilité « in vitro » des aliments testés. Ainsi, NEATHERy (1969) distribue à des bœufs du foin à 7,4 ou 18,8 p. cent de matières azotées et observe pour cinq fourrages différents des coefficients de digestibilité « in vitro» plus élevés lorsque la ration contient le foin riche en matières azotées : en moyenne les coefficients sont augmentés de 15,8 p. cent. De même, BezEAu (1965) distribue à deux vaches donneuses de liquide de rumen cinq rations constituées de foins aux teneurs en matières azotées et en cellulose différentes (de 6,7 à 18 p. cent pour la matière azotée, de 37,1 à 26,2 p. cent pour la cellulose). De manière surprenante, il enregistre des coefficients de digestibilité de la cellulose plus élevés avec les foins à teneur faible en cellulose et à teneur élevée en matières azotées.

L'absence de différences significatives attribuables aux trois rations peut paraître étonnante alors que le choix d'aliments de nature ou de conditionnement différents suffit dans certains cas à modifier l'activité du liquide de rumen et les digestibilités «in vitro». Ainsi, Figroid, Hale \& Theurer (1972) qui distribuent à des bœufs des rations à base de foins de luzerne, d'orge et de sorgho observent, par la technique des sachets de nylon, des coefficients de digestibilité de la matière sèche des grains de sorgho et d'orge plus élevés avec la ration contenant de la luzerne. CaLder (1970) \& ClaRK (1975) distribuent à des animaux fistulisés du foin seul ou du foin complémenté par des céréales. Ils enregistrent les meilleurs coefficients de digestibilité « in vitro » lorsque les animaux reçoivent la ration constituée du foin seul. En revanche, dans un régime à $14,5 \mathrm{p}$. cent de matières azotées distribué à des bœufs, BAILEY \& HironaKa (1970) font varier les proportions d'orge et de luzerne; ils observent que les coefficients de digestibilité en sachets de l'orge et de la luzerne utilisés dans la ration tendent à augmenter lorsque la proportion d'orge s'élève.

Orskov \& MeHrez (1975) montrent que le conditionnement de l'aliment dans la ration (orge entière ou moulue et condensée) influence de manière hautement signi- 
ficative les résultats des tests de digestibilité en sachets de nylon de l'herbe séchée : après 24 heures d'incubation, le coefficient de digestibilité de la matière sèche est de $62,5 \mathrm{p}$. cent lorsque les moutons reçoivent de l'orge entière et seulement de 42,3 p. cent lorsqu'ils reçoivent de l'orge condensée.

Knipfel \& Troelsel (1966) notent également un effet de la ration sur les coefficients de digestibilité qui s'estompe cependant lorsque la durée d'incubation passe de 12 à 48 heures. Dans nos expériences, la durée d'incubation de 48 heures a peutêtre atténué l'effet des régimes : seule une étude de la cinétique de la réaction aurait pu mettre en évidence l'effet de la durée d'incubation. Par contre, les différences individuelles que nous avons enregistrées pour les coefficients « in vitro » de la matière sèche et de la matière organique sont très hautement significatives et en accord avec les résultats signalés par BEzEAU (1965) ainsi que par Nelson et al. (1972).

Les déterminations «in vitro» destinées à prévoir la digestibilité d'un aliment ne sont pas des techniques absolues comme pourraient l'être les déterminations " in vivo». De multiples facteurs sont susceptibles de faire varier les résultats obtenus avec ces techniques; c'est pourquoi, pour obtenir des résultats reproductibles entre laboratoires, il serait hautement souhaitable de standardiser soigneusement les conditions expérimentales.

Accepté pour publication en mai 1981.

\section{Remerciements}

Travail réalisé avec l'aide financière de l'Institut pour l'Encouragement de la Recherche Scientifique dans l'Industrie et l'Agriculture (I.R.S.I.A.).

\section{Summary}

In vitro determination of digestibility of hay treated with $\mathrm{NaOH}$ or untreated

The Tilley and Terry method and nylon bag technique were used to determine the digestibility of hay treated or untreated with $\mathrm{NaOH}$ using a farm machine. The $\mathrm{NaOH}$ concentrations were 3 and $6 \%$ on a feed base.

Twelve young bulls receiving 3 rations differing by the proportions of the feed components were used as donors for rumen liquid as well as carrier animals for the nylon bags. The applied digestibility techniques demonstrate the effectiveness of the $\mathrm{NaOH}$ treatment but they clearly overestimate the $\mathrm{NaOH}$ action as compared to the «in vivo » digestibility. The highest \& in vitro» digestibility was observed with the $3 \% \mathrm{NaOH}$ treatment. The composition of the rations apparently did not affect the «in vitro digestibility coefficients. However, between animals statistically significant differences were observed. 


\section{Références bibliographiques}

Aerts J.V., Debrabander D.L., Cottyn B.G., Buysse F.X., 1976. Contribution à la détermination de la valeur alimentaire des fourrages grossiers. III. Estimation de la digestibilité et de la valeur énergétique par la méthode des sachets de nylon. Revue agric. Brux., 29, 255-268.

Ali C.S., Mason V.C., Waagepetersen J., 1977. The voluntary intake of pelleted diets containing sodium hydroxide-treated wheat straw by sheep. I. The effect of the alkali concentration in the straw. Z. Tierphysiol. Tiernähr. Futtermillelk, 39, 173-182.

Anderson D.C., Ralston A.T., 1973. Chemical treatment of rye-grass straw : "in vitro» dry matter digestibility and compositional changes. J. anim. Sci., 37, 148-152.

Bailey C.B., Hironaka R., 1970. Maximum loss of feed from nylon bags in the rumens of steers as related to apparent digestibility. Can. J. anim. Sci., 50, 325-330.

Berger L., Klopfenstein T., Britton R., 1979. Effect of sodium hydroxide on efficiency of rumen digestion. J. anim. Sci., 49, 1317-1323.

BezEAU L.M., 1965. Effect of source of inoculum on digestibility of substrate in «in vitro» digestion trials. J. anim. Sci., 24, 823-825.

Calder F.W., 1970. Effect of barley supplement to the ration of donor animals used in the «in vitro» digestibility determination. Can. J. anim. Sci., 50, 265-267.

Clark J., 1975. "In vitro» digestion of mixtures of grain and hay. Austr. J. exp. Agric. anim. Husb., 15, 471-474.

CoOmbe J.B., Dinius D.A., WheEler W.E., 1979. Effect of alkali treatment on intake and digestion of barley straw by beef steers. J. anim. Sci., 49, 169-176.

Cottyn B.G., Van Hee L.P., Carlier L.A., 1970. La détermination de la digestibilité et de la valeur nutritive des fourrages bruts au moyen de la technique de digestibilité « in vitro». Revue agric. Brux., 23, 1491-1504.

Dagnælie P., 1970. Théorie et méthodes statistiques II. 451 pages. Ed. Duculot-Gembloux.

Dulphy I.-P., 1979. Valeur alimentaire des pailles traitées ou non. Bull. tech. Inf., 341342, 319-335.

Figroid W., Hale W.H., Theurer B., 1972. An evaluation of the nylon bag technique for estimating rumen utilization of grains. J. anim. Sci., 35, 113-120.

Knipfel J.E., Troelsen J.E., 1966. Interaction between inoculum donor diet and substrate in « in vitro» ruminant digestion studies. Can. J. anim. Sci., 46, 91-95.

Levy D., Holzer Z., Neumark H., Folman Y., 1977. Chemical processing of wheat straw and cotton by-products for fattening cattle. I. Performance of animals receiving the wet material shortly after treatment. Anim. Prod., 25, 27-37.

Mwakatundu A.G.K., OWen E., 1974. «In vitro» digestibility of sodium hydroxide-treated grass harvested at different stages of growth. East afr. agric. for. J., 40, 1-10.

NEATHERY M.W., 1969. Dry matter disappearance of roughages in nylon bags suspended in the rumen. J. Dairy Sci., 5z, 74-78.

Nelson B.D., Ellezy H.D., Montgomery C., Morgan E.B., 1972. Factors affecting the variability of an «in vitro» rumen fermentation technique for estimating forage quality. J. Dairy Sci., 55, 358-366.

Nik-Khah A., Tribe D.E., 1977. A note on the effect of diet on the inoculum used in digestibility determination «in vitro». Anim. Prod., 25, 103-106.

Orskov E.R., Mehrez A.Z., 1975. The effect of feeding with whole or pelleted barley on rate of digestion of dried grass in the rumen of sheep. Proc. Nutr. Soc., 34, 64 A-65 A.

Rexen F., Thomson K., 1976. The effect on digestibility of a new technique for alkali treatment of straw. Anim. Feed Sci. Technol., 1, 73-83.

SChNeIder B.W., FLatT W.P., 1975. The evaluation of feeds through digestibility experiments. The University of Georgia Press, Athens, 165-167. 
SingH M., JACKSON M.G., 1971. The effect of different levels of sodium hydroxide spray treatment of wheat straw on consumption and digestibility by cattle. J. agric. Sci. Camb., 77, 5-10.

Tilley J.M.A., Terry R.A., 1963. A two-stage technique for the «in vitro» digestion of forage crops. J. Br. Grassld. Soc., 18, 104-111.

Van Eenaeme C., Istasse L., Lambot O., Bienfait J.M., Gielen M., 1981. Effect of sodium hydroxide treatment on chemical composition, «in vivo» and «in vitro» digestibility of hay. Agriculture and Environment (To be published).

Van HeE L.P., Carlier L.A., 1970. Bepaling van de «in vitro» verteerbaarheid van groenvoedergewassen. Medeling R.V.P., $\mathrm{n}^{\circ}$ 347. Rijksstation voor Plantenveredeling. Merelbeke (Belgium).

WILson R.K., PIgDen W.J., 1964. - Effect of sodium hydroxide treatment on the utilization of wheat straw and poplar wood by rumen micro organisms. Can. J. anim. Sci., 44, 122-123. 